Ebisu Ebisu

Études japonaises Études japonaises

50 | automne-hiver 2013

Création et valeurs dans le Japon moderne

\title{
Pierre-François SOUYRI, Nouvelle Histoire du Japon
}

Paris, Perrin, 2010, 622 pages.

Laurent Nespoulous

\section{OpenEdition}

1 Journals

Édition électronique

URL : http://journals.openedition.org/ebisu/1240

DOI : 10.4000/ebisu. 1240

ISSN : 2189-1893

Éditeur

Institut français de recherche sur le Japon à la Maison franco-japonaise (UMIFRE 19 MEAE-CNRS)

Édition imprimée

Date de publication : 1 octobre 2013

Pagination : 229-232

ISSN : 1340-3656

\section{Référence électronique}

Laurent Nespoulous, «Pierre-François sourri, Nouvelle Histoire du Japon », Ebisu [En ligne], 50 | automne-hiver 2013, mis en ligne le 21 juin 2014, consulté le 10 décembre 2020. URL : http:// journals.openedition.org/ebisu/1240 ; DOI : https://doi.org/10.4000/ebisu.1240

Ce document a été généré automatiquement le 10 décembre 2020.

(c) Institut français de recherche sur le Japon à la Maison franco-japonaise 


\title{
Pierre-François SOUYRI, Nouvelle Histoire du Japon
}

Paris, Perrin, 2010, 622 pages.

\author{
Laurent Nespoulous
}

\section{RÉFÉRENCE}

Pierre-François SOUYRI, Nouvelle Histoire du Japon, Paris, Perrin, 2010, 622 pages.

1 Peu nombreux sont les livres présentant, en français, une histoire générale du Japon. Il convient donc de saluer toute nouvelle tentative éditoriale synthétique en la matière. La Nouvelle Histoire du Japon de Pierre-François Souyri est la plus récente publication de ce type, portant à trois les ouvrages de ce genre, réalisés par des historiens français, chacun étant, dans son format, fort différent des deux autres. Il y a tout d'abord : 1) le "Que sais-je? » de Michel Vié, Histoire du Japon des origines à Meiji, régulièrement révisé et réédité depuis 1973 ; 2) le livre collectif dirigé par Francine Hérail, Histoire du Japon des origines à Meiji (auquel a d'ailleurs participé Pierre-François Souyri), publié aux Éditions Horvath en 1990, revu et augmenté aux Éditions Hermann, en 2010, sous le titre Histoire du Japon des origines à nos jours ; 3) et enfin, publié également en 2010 , le présent volume. La Nouvelle Histoire du Japon est une contribution originale en ce sens qu'il s'agit du fruit de l'esprit de synthèse d'un seul auteur, développé en un volume de plus de 600 pages.

2 Trois années se sont déjà écoulées depuis la parution de la première version de ce travail, laquelle a été fort bien accueillie par un public notamment étudiant avide de grandes synthèses claires. Rendre compte d'une telle somme n'est pas aisé, du fait même de son ampleur diachronique. Il conviendra donc ici plutôt d'évaluer, si cela peut nous être permis, "l'efficacité » de l'ensemble. Lorsqu'un seul et unique auteur entreprend un travail de synthèse aussi important que celui-ci, se transformant ainsi en quelque sorte en « homme-orchestre ", il est bien évident que la tâche ne va pas sans péril. Dès les premières pages, l'auteur met en garde le lecteur (p. 9) : son ouvrage étant 
le récit chronologique de l'histoire générale de l'archipel, il a bien entendu été nécessaire de faire des choix, tant sur le plan thématique, que, ajouterons-nous, narratif et factuel.

3 Si le récit est certes organisé selon une trame chronologique, il est presque avant tout thématique. À l'intérieur des grands cadres que sont la Préhistoire et la Protohistoire, l'Antiquité, le Moyen Âge, l'époque Moderne et l'époque Contemporaine, PierreFrançois Souyri a décidé de mettre l'accent sur tel ou tel élément qui fait historiquement sens. Ce travail de synthèse n'a donc rien d'événementiel, et c'est ce qui lui garantit un fort potentiel pédagogique. À la périodisation classique se superposent donc surtout de grands sujets comme, par exemple, le peuplement de l'archipel (p.11-32), la formation des premières élites (p. 33-68), la construction d'un nouveau système politique aux époques archaïque et antique (p.69-133), la formulation de l'aristocratie guerrière et de son pouvoir au Moyen Âge (p. 187-310), la fondation d'un "Ancien Régime » (p. 353-387) à l'époque Moderne et la construction de l'État-nation (p. 443-477) à partir de l'ère Meiji, et ainsi de suite, jusqu'à nos jours. Se succèdent ainsi des thèmes sur lesquels l'historiographie japonaise s'est montrée foisonnante.

4 Nous relèverons trois grandes qualités dans cet ouvrage. L'une réside en son plan facile à suivre, progressif, qui peut certes parfois laisser à penser que les enchaînements de l'histoire sont mécaniques, mais qui permet de restituer une histoire claire, regroupée autour de faits importants pour la compréhension générale de l'archipel au cours des 2000 dernières années. Une autre renvoie à l'image que l'on se fait de l'histoire japonaise en France. En effet, l'ouvrage laisse appréhender un niveau de complexité en principe rarement présent dans les manuels d'histoire. Cela permet, notamment, de sortir de certains poncifs (le Japon guerrier, éternel empire totalitaire et colonialiste, par exemple) dont est victime l'histoire du Japon en France. Enfin, étant nous même archéologue, la troisième grande qualité que nous tenons à souligner tient au fait que sont régulièrement convoqués (pour les périodes d'« avant l'histoire », de l'Antiquité, mais également pour le Moyen Âge), les résultats émanant d'une source nouvelle de la construction de l'histoire : l'archéologie. L'archéologie a une longue histoire au Japon, mais ce n'est que depuis quelques décennies que l'on tient réellement compte de ses résultats dans la validation, ou l'invalidation, des sources textuelles, sans oublier son importance quand ces dernières font justement défaut. On ne peut que se féliciter de l'intégration de cette historiographie.

5 Tout cela donne un aperçu du grand dynamisme de la recherche historique au Japon ${ }^{1}$. Un regret que l'on peut toutefois formuler - très probablement dû à des limitations posées par l'éditeur-, porte sur le manque de références plus directes aux sources japonaises, lesquelles affleurent pourtant sans ambiguïté sous la plume de l'auteur. La bibliographie par chapitre, en fin d'ouvrage, ne viendra pas combler ce manque, qui peut s'avérer dommageable aux étudiants qui chercheraient des pistes de lecture en langue japonaise. L'outil pédagogique que constitue donc cet ouvrage trouve ici une de ses limites. Une autre réserve qu'il nous semble légitime de formuler est relative à la structure interne des différentes parties de l'ouvrage. Si le plan est bien chronologique et thématique, en revanche, les thèmes abordés ne se répondent pas tous d'un chapitre à l'autre. Un exemple : les chapitres portant sur le Moyen Âge, spécialité de PierreFrançois Souyri, abordent, à de multiples reprises, le rapport des élites à la société, à la terre et à ceux qui la cultivent, les paysans (l'auteur marque même les esprits en reprenant dans une de ses sous-parties le titre de l'ouvrage de Georges Duby², 
"Guerriers et Paysans ", p. 244). Mais dans les autres grandes parties, ce thème est relativement sous-représenté, notamment pour l'Antiquité, et les périodes la précédant, lesquelles voient pourtant se former les premières sociétés de paysans de l'archipel. Inversement, une attention est légitimement portée à la formation des élites de l'archipel, en contexte antique et sub-antique, mais dans la suite de l'ouvrage, cette thématique n'est présente qu'aléatoirement, en fonction des époques, alors que «des élites", anciennes ou nouvelles, sont toujours, bien sûr, présentes tout au long de l'histoire. L'ouvrage manque donc, en ce sens, de constance dans le traitement de thèmes qui mériteraient d'être abordés à chaque étape. La critique n'est pas majeure, mais il est vrai que l'on perd la trace de certaines problématiques d'une époque à une autre, et dans la mesure où le récit historique demeure fluide, cela peut donner au lecteur l'impression que certains sujets cessent d'être d'actualité, alors qu'il s'agit d'un effet lié au choix de focale dans la narration.

6 Au terme de la lecture de l'ouvrage de Pierre-François Souyri, une conclusion s'impose, hors de tout doute : il s'agit bien là d'un livre dont la lecture ne peut qu'être fortement recommandée à tous ceux qui souhaitent se constituer une image d'ensemble de l'histoire de l'archipel japonais. Si ce travail est justiciable de certaines critiques, ces dernières tendent à relever, à notre sens, des « défauts de ses qualités ».

7 Forts de trois ouvrages d'histoire générale disponibles en français ${ }^{3}$, il nous faut désormais appeler de nos vœux la réalisation d'une plus vaste et détaillée histoire du Japon, grand travail d'équipe qui permettrait de s'affranchir des limites éditoriales qu'impose l'écriture d'un seul ouvrage, et qui embrasserait alors l'ensemble de l'histoire et de l'historiographie du Japon.

\section{NOTES}

1. Hormis l'archéologie, discipline de pointe en France tout comme au Japon, on peut plus généralement faire remarquer que le Japon est « en avance » sur certains sujets par rapport à la recherche hexagonale, par exemple, très tôt à partir des années 1960, en histoire de la colonisation.

2. Georges Duby, Guerriers et Paysans (VII-XII siècle). Premier essor de l'économie européenne, Paris, Gallimard, 1974.

3. Nous laissons de côté l'ouvrage d'Edwin Reischauer, Histoire du Japon et des Japonais, Paris, Seuil, édition révisée de 1973, 2 vol., déjà fort ancien, certes très facile d'accès, qui n'est pas à jour des résultats de la recherche japonaise. 


\section{AUTEURS}

LAURENT NESPOULOUS

Umifre $19 \mathrm{MFJ}$ 